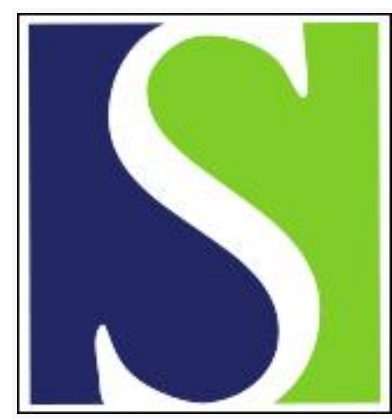

Scand J Work Environ Health 2006;32(3):204-208

https://doi.org/10.5271/sjweh.1000

Issue date: 30 Jun 2006

Prevalent cardiovascular disease, risk factors and selection out of shift work

by Kivimäki $M$, Virtanen $M$, Elovainio $M$, Väänänen $A$, Keltikangas-Järvinen L, Vahtera J

Affiliation: Finnish Institute of Occupational Health, Topeliuksenkatu 41 a A, FIN-00250 Helsinki, Finland. mika.kivimaki@ttl.fi

Refers to the following texts of the Journal: 1999;25(2):100-104 1999;25(2):85-99 1997;23(4):257-265

The following articles refer to this text: 2008;34(3):206-212;

2009;35(3):163-179; 2009;35(3):157-161; 2009;35(5):361-367; 2011;37(5):402-410; 2012;38(4):343-348; 2014;40(3):287-294

Key terms: cardiovascular disease; risk factor; selection bias; shift work

This article in PubMed: www.ncbi.nlm.nih.gov/pubmed/16804623

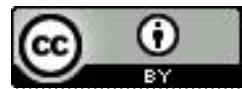




\title{
Prevalent cardiovascular disease, risk factors and selection out of shift work
}

\author{
by Mika Kivimäki, PhD, ${ }^{1,2}$ Marianna Virtanen, PhD, ${ }^{1}$ Marko Elovainio, PhD, ${ }^{3}$ Ari Väänänen, PhD, ${ }^{1}$ Liisa \\ Keltikangas-Järvinen, PhD, ${ }^{2}$ Jussi Vahtera, $P h D^{1}$
}

\begin{abstract}
Kivimäki M, Virtanen M, Elovainio M, Väänänen A, Keltikangas-Järvinen L, Vahtera J. Prevalent cardiovascular disease, risk factors and selection out of shift work. Scand J Work Environ Health 2006;32(3):204-208.
\end{abstract}

\begin{abstract}
Objectives This study examines whether health-related selection out of shift work is likely to bias the association between shift work and cardiovascular disease (CVD).

Methods Survey data on doctor-diagnosed CVD (myocardial infarction, angina, or hypertension) and risk factors (high blood pressure, high cholesterol concentration, obesity, and diabetes) were collected in 2000-2002 for a cohort of 7037 female nurses (5038 shift workers, 1999 day workers) in 21 Finnish hospitals. The followup in 2004 determined those who had left their workplace or changed from shift work to day work.

Results Among the shift workers, the age-adjusted odds for leaving the organization was 1.83 (95\% CI 1.013.32) times higher for those with prevalent diabetes and 2.21 (95\% CI 1.12-4.39) times higher for those with three to four risk factors than for their counterparts with no diabetes or risk factors. The associations between CVD risk factors and leaving the organization were similar for the day workers. The prevalent CVD and risk factors did not predict a change to day work among the shift workers who remained in the organization during the entire follow-up period.

Conclusions Employees with several risk factors are more likely to leave an organization regardless of the type of work schedule. Health-related selection out of shift work is an unlikely source of major bias in research on shift work and CVD.
\end{abstract}

Key terms cardiovascular disease; risk factor; selection bias; shift work.

Observational studies have suggested that shift work is associated with a higher risk of cardiovascular disease (CVD) than day work, but the risk estimates have varied between studies (1-9). For example, in a large study of nurses in the United States and a study of Finnish workers the relative risk of coronary heart disease was $1.5(4,5)$, whereas, in a Danish investigation, mortality from ischemic heart disease did not differ between shift and day workers (9). In addition to potential confounding by third factors that relate to both shift work and CVD (eg, socioeconomic position) (9), both selection bias and reversed causality may contribute to imprecise estimations based on observational data (5). As stronger study designs, such as randomized controlled trials, may be unfeasible or unethical in this context, assessing the magnitude of bias in observational studies would be essential to the proper interpretation of shiftwork effects.

The healthy worker effect is a common source of selection bias, and it occurs when healthier workers are selected for work and those with health problems drop out $(10,11)$. A healthy worker effect that is stronger for shift workers than for day workers would bias the relative risk of CVD for shift work towards the null, whereas an opposite case is likely to lead to an overestimation of the adverse effect of shift work (5). Within the working population, a large proportion of employees changes the work schedule over time $(6,12)$, and this change can create additional selection bias through reversed causality between shift work and CVD. For example, if shift workers with prevalent CVD or a high CVD risk profile are more likely to change to day work than other shift workers are, then the observed associations between shift work and CVD are likely to underestimate the actual impact of shift work on CVD.

Many studies on shift work and CVD have acknowledged the possibility of selection bias when results are interpreted $(5,9,13)$, but few have directly examined whether these selective processes indeed differ between

1 Finnish Institute of Occupational Health, Helsinki, Finland.

2 University of Helsinki, Department of Psychology, Helsinki, Finland.

3 National Research and Development Centre for Welfare and Health, Helsinki, Finland.

Reprint requests to: Dr M Kivimäki, Finnish Institute of Occupational Health, Topeliuksenkatu 41 a A, FIN-00250 Helsinki, Finland. [E-mail: mika.kivimaki@ttl.fi] 
shift and day workers and cause a significant threat with respect to validity. In this prospective study, we examined this issue by determining the extent to which prevalent CVD and its risk factors are predictive of a person leaving an organization and the extent to which they predict change to day work among shift workers. To minimize confounding by socioeconomic position (9), we limited the analyses to one occupational group, nurses.

\section{Study population and methods}

\section{Study population}

This study is part of the on-going Finnish hospital personnel study, in which the baseline questionnaire survey was sent to all 21938 employees working in 21 Finnish hospitals in 2000-2002 (the eligible population). Altogether 15346 employees (70\%) responded to this survey, and, of them, 11976 were also working in the target hospitals 2 to 4 years later at the time of the follow-up in 2004. Of these, 9845 (82\%) responded to the follow-up survey. The gender and age differences between the respondents and the eligible population were small. Among the follow-up survey respondents, women comprised $88 \%$, and the mean age at baseline was 41 years. For the eligible population, the corresponding figures were $84 \%$ and 43 years. This study focused on the largest single occupational group and women only (ie, the 7037 female nurses 20 to 63 years of age who indicated their shift schedule in the baseline survey) (table 1).

\section{Baseline measures}

Shift work and day work were measured by asking the respondents to rate which of the following categories described their current shift schedule: (i) permanent day shift, (ii) three-shift schedule, (iii) two-shift schedule, and (iv) permanent night shift (14). The first category was considered day work, and the other three categories comprised shift work.

CVD was measured with a self-administered checklist of common chronic conditions $(15,16)$. The participants were asked the following question: "Have you ever been told by a physician that you have or have had any of the following diseases?" A list of common conditions with response options "yes" or "no" after each condition followed this question. Prevalent CVD was determined by affirmative responses on "myocardial infarction", "angina pectoris", or "hypertension". The agreement between these self-assessments and data from medical records has been shown to be substantial for these specific medical conditions (kappa 0.73-0.80) in a representative sample of Finnish men and women (15, 16). Since our sample was comprised exclusively of health professionals, the accuracy of self-reported CVD may have been higher than that found in a general population

The following four cardiovascular risk factors were assessed: elevated blood pressure, high cholesterol, obesity, and diabetes. Therefore information on elevated blood pressure, high cholesterol, and diabetes was requested with the same self-administered checklist of common chronic conditions as CVD $(15,16)$. Self-reported weight and height were used to measure body mass index (BMI, $\mathrm{kg} / \mathrm{m}^{2}$ ), and a BMI of $\geq 30.0 \mathrm{~kg} / \mathrm{m}^{2}$ was used to identify obesity. All of the risk factor measures showed high predictive validity for our data. Among those with no CVD at baseline, the age-adjusted odds ratio for incident CVD by follow-up was 11.85 (95\% CI 8.60-16.32) times higher among those with an elevated baseline blood pressure than among those with normal blood pressure. The corresponding odds ratio was 1.68 (95\% CI 1.13-2.50) for high cholesterol, 2.00 (95\% CI 0.70-5.75) for diabetes, and 2.39 (95\% CI 1.61-3.54) for obesity at baseline.

\section{Follow-up measures}

The two dichotomous outcome variables were leaving the organization by the time of the follow-up (no; yes) and change to day work by the time of follow-up (no; yes). We identified all of the baseline respondents who were still in the service of the target hospitals at the time of the follow-up survey using employers' records. The baseline respondents who were not in the records were categorized as having left the organization (no data were

Table 1. Characteristics of the female nurses. (CVD = cardiovascular disease)

\begin{tabular}{|c|c|c|c|}
\hline \multirow[t]{2}{*}{ Characteristic } & $\begin{array}{l}\text { Shift workers } \\
\text { at baseline } \\
(\mathrm{N}=5038)\end{array}$ & $\begin{array}{c}\text { Day workers } \\
\text { at baseline }{ }^{b} \\
(\mathrm{~N}=1999)\end{array}$ & \multirow[t]{2}{*}{$\begin{array}{c}\text { P-value } \\
\text { for } \\
\text { difference } \\
c, d\end{array}$} \\
\hline & $\%$ & $\%$ & \\
\hline
\end{tabular}

\begin{tabular}{|c|c|c|c|c|c|}
\hline \multicolumn{6}{|l|}{ Baseline } \\
\hline Prevalent CVD e & 4964 & 9.1 & 1949 & 11.6 & 0.74 \\
\hline High blood pressure & 4927 & 14.2 & 1940 & 17.3 & 0.54 \\
\hline High cholesterol level & 4922 & 11.0 & 1934 & 14.2 & 0.87 \\
\hline Obesity & 4941 & 9.6 & 1975 & 8.5 & 0.004 \\
\hline Diabetes & 4920 & 1.3 & 1926 & 2.0 & 0.12 \\
\hline \multicolumn{6}{|l|}{ Follow-up } \\
\hline Leaving the organization & 5038 & 23.6 & 1999 & 22.8 & 0.99 \\
\hline Change to day work ${ }^{\dagger}$ & 3221 & 8.1 & - & - & - \\
\hline
\end{tabular}

a The mean age for the shift workers was 41.1 (SD 9.5) years.

${ }^{\mathrm{b}}$ The mean age for the day workers was 45.3 (SD 8.6) years.

c $P<0.0001$.

${ }^{d}$ Analysis of variance for mean age, age-adjusted logistic regression analysis for other variables.

e Self-reported physician-diagnosed myocardial infarction, angina, or hypertension.

${ }^{f}$ Among the baseline shift workers who remained in the organization. 
available on their employment status or shift mode after baseline). Information on change to day work by the time of the follow-up involved only those who responded both at baseline and at follow-up. The follow-up survey indicated work schedules with a question similar to that included in the baseline survey. We divided those who reported working in shifts at baseline into those who stayed in shift work (respondents who reported shift work at follow-up) and those who changed to day work (respondents who reported day work at follow-up).

\section{Statistical analysis}

We assessed the difference in the mean age between the shift workers and day workers with an analysis of variance. The differences in prevalent CVD and its risk factors (all binary variables) between these groups were examined with logistic regression models. Odds ratios and $95 \%$ confidence intervals were adjusted for age in 10 -year categories $(<25,25-34,35-44,45-54, \geq 55$ years). The age-adjusted associations of prevalent CVD and its risk factors with leaving the organization were assessed with separate logistic regression models for the shift workers and day workers. An additional analysis examined whether the number of risk factors $(0,1,2$ or 3-4) was predictive of leaving the organization. The differences in these associations between the groups were examined for the entire cohort with age-adjusted logistic models including prevalent CVD or a risk factor, work schedule at baseline (shift work versus day work) and the interaction term between prevalent CVD or risk factor and work schedule as independent variables. A statistically significant interaction term was taken as an indication that the associations between CVD or a risk factor and leaving the organization differed between the shift workers and day workers. Finally, we calculated age-adjusted logistic regression models to examine the associations between prevalent CVD and its risk factors and changing to day work among those who worked shifts at baseline. All of the data analyses were conducted using the SAS software package (SAS Institute, Cary, $\mathrm{NC}, \mathrm{USA}$ ).

\section{Results}

Table 1 shows the characteristics of the study population. Among the shift workers, $81 \%$ reported working a three-shift schedule, $15 \%$ had a two-shift schedule, and $4 \%$ worked a permanent night shift. The shift workers were an average of 4 years younger than the day workers. After adjustment for age, the prevalence of obesity was slightly higher among the shift workers than among the day workers, but no group differences were found in relation to $\mathrm{CVD}$, its risk factors, or the proportion of employees who left the organization by the time of the follow-up.

Table 2 presents the age-adjusted associations between prevalent CVD and its risk factors and the likelihood of

Table 2. Prevalent cardiovascular disease and risk factors as predictors of leaving the organization among the female nurses. The odds ratios (OR) and $95 \%$ confidence intervals $(95 \% \mathrm{Cl})$ have been adjusted for age.

\begin{tabular}{|c|c|c|c|c|c|c|c|c|}
\hline \multirow[t]{2}{*}{ Baseline characteristic } & \multicolumn{4}{|c|}{ Shift workers at baseline } & \multicolumn{4}{|c|}{ Day workers at baseline } \\
\hline & Total (N) & Cases (N) & $\mathrm{OR}$ & $95 \% \mathrm{Cl}$ & Total (N) & Cases (N) & OR & $95 \% \mathrm{Cl}$ \\
\hline \multicolumn{9}{|l|}{ Cardiovascular disease } \\
\hline No & 4511 & 1058 & 1.00 & . & 1723 & 370 & 1.00 & . \\
\hline Yes & 453 & 109 & 1.04 & $0.80-1.36$ & 226 & 76 & 1.53 & $1.08-2.17$ \\
\hline \multicolumn{9}{|l|}{ High blood pressure } \\
\hline No & 4227 & 970 & 1.00 & . & 1605 & 345 & 1.00 & . \\
\hline Yes & 700 & 184 & 1.14 & $0.93-1.40$ & 335 & 98 & 1.29 & $0.96-1.73$ \\
\hline \multicolumn{9}{|l|}{ High cholesterol } \\
\hline No & 4382 & 1011 & 1.00 & . & 1660 & 352 & 1.00 & . \\
\hline Yes & 540 & 137 & 1.06 & $0.83-1.35$ & 274 & 83 & 1.29 & $0.93-1.80$ \\
\hline \multicolumn{9}{|l|}{ Obesity } \\
\hline No & 4466 & 1069 & 1.00 & . & 1807 & 397 & 1.00 & . \\
\hline Yes & 475 & 104 & 0.93 & $0.72-1.19$ & 168 & 49 & 1.31 & $0.88-1.93$ \\
\hline \multicolumn{9}{|l|}{ Diabetes } \\
\hline No & 4858 & 1128 & 1.00 & . & 1888 & 426 & 1.00 & . \\
\hline Yes & 62 & 23 & 1.83 & $1.01-3.32$ & 38 & 12 & 1.62 & $0.76-3.46$ \\
\hline \multicolumn{9}{|l|}{ Number of risk factors } \\
\hline 0 & 3472 & 806 & 1.00 & . & 1307 & 263 & 1.00 & . \\
\hline 1 & 967 & 225 & 1.01 & $0.84-1.22$ & 420 & 104 & 1.24 & $0.93-1.65$ \\
\hline 2 & 277 & 67 & 0.99 & $0.71-1.37$ & 114 & 37 & 1.44 & $0.90-2.30$ \\
\hline $3-4$ & 48 & 20 & 2.21 & $1.12-4.39$ & 36 & 15 & 2.24 & $1.03-4.87$ \\
\hline
\end{tabular}


leaving the organization during the follow-up. Among the shift workers, diabetes and a clustering of three to four risk factors, but not the other baseline characteristics, were associated with an increased likelihood to leave the organization. Among the day workers, the associations were similar to those found for the shift workers, and the interactions between the shiftwork schedule status and prevalent CVD or its risk factors and leaving the organization were all statistically nonsignificant ( $\mathrm{P}=0.87$ for $\mathrm{CVD}, \mathrm{P}=0.90$ for high blood pressure, $\mathrm{P}=0.25$ for obesity, $\mathrm{P}=0.90$ for cholesterol, $\mathrm{P}=0.72$ for diabetes, and $\mathrm{P}=0.83$ for a clustering of three or four risk factors).

Table 3 shows the age-adjusted associations between prevalent CVD and its risk factors and change to day work during the follow-up among the shift workers at baseline who did not leave the organization. All of these associations were nonsignificant. [However, note that, as none of the diabetic employees changed to day work, this variable was not entered into the analysis.]

\section{Discussion}

This study found no evidence of a selection bias or reversed causality that could significantly under- or overestimate the relative risk of shift work for CVD. Prevalent CVD, elevated blood pressure, high cholesterol concentration, obesity, and diabetes at baseline were equally predictive of leaving the organization among the shift workers and day workers. These medical conditions and risk factors were not associated with a subsequent change from shift work to day work. Our study was based on a prospective design and a large contemporary sample of female employees. The inclusion of only one occupational group minimized potential confounding by socioeconomic differences between the shift workers and day workers.

A recent review of 17 studies on shift work and CVD concluded that most of the large studies reported a risk estimate of around 1.4, but that four large studies failed to find an association (1). Health-related selection out of shift work was suggested as one of the potential explanations for null findings, and indirect support for this possibility is provided by a British study that found higher CVD risk among ex-shift workers than among current shift workers (3). However, this empirical evidence was related to a historical cohort that worked in 1956-1968. Selection data for our cohort were collected in 2000-2004, and, on the basis of this more contemporary evidence, no major differences in CVD or its risk factors were evident between those who changed from shift work to day work and those who remained in
Table 3. Prevalent cardiovascular disease and risk factors as predictors of change to day work among the female nurses with shift work. The odds ratios (OR) and 95\% confidence intervals $(95 \% \mathrm{Cl})$ have been adjusted for age.

\begin{tabular}{lrrrc}
\hline & Total (N) & Cases (N) & OR & $95 \% \mathrm{Cl}$ \\
\cline { 2 - 5 } Cardiovascular disease & & & & \\
$\quad$ No & 2897 & 232 & 1.00 &. \\
$\quad$ Yes & 285 & 24 & 1.15 & $0.74-1.81$ \\
High blood pressure & & & & \\
$\quad$ No & 2739 & 216 & 1.00 &. \\
$\quad$ Yes & 427 & 34 & 1.04 & $0.71-1.53$ \\
High cholesterol & & & & \\
No & 2824 & 228 & 1.00 &. \\
Yes & 336 & 23 & 0.89 & $0.57-1.41$ \\
Obesity & & & & \\
No & 2855 & 235 & 1.00 &. \\
Yes & 303 & 22 & 0.88 & $0.56-1.38$ \\
Diabetes & & & & \\
No & 3128 & 253 & NA &. \\
Yes & 32 & 0 & NA & $\cdot$ \\
Number of risk factors & & & & \\
0 & 2251 & 189 & 1.00 &. \\
1 & 615 & 38 & 0.73 & $0.51-1.05$ \\
2 & 170 & 14 & 1.07 & $0.60-1.91$ \\
$3-4$ & 24 & 2 & 1.08 & $0.25-4.65$ \\
\hline
\end{tabular}

shift work. The change in work status among the female nurses seemed to be determined by factors that are not strongly associated with cardiovascular health.

We assessed prevalent CVD and its risk factors with validated self-report measures, and such an assessment reflects only medical conditions and risks that the shift workers were fully aware of. Effects of self-reported diseases on career choices (leaving the organization or changing from shift work to day work) may be as strong as or stronger than those obtained from objectively examined medical conditions and risk factors, because the latter measurements would also include diseases and risk levels that the employees are not aware of (eg, undiagnosed hypertension) and they may affect the planning of a career less than diseases and risks that the workers recognize. Considering that no evidence of selection bias or reversed causality was obtained with the selfreported assessments, we assumed that replications with objectively assessed CVD and risk factors would lead to null findings as well.

While our study covers selection out of shift work, it is important to recognize that the selection process may also operate at the time of entrance into shift work. When people make a decision to apply for shift work, they have probably estimated their ability to withstand irregular workhours and may therefore differ in terms of health from those choosing not to apply. Moreover, workplaces may use different hiring criteria when selecting shift workers than they do for day workers. It has been suggested that this primary selection would 
lead to an underestimation of the true CVD risk in relation to shift work (1), but, to our knowledge, there is no direct empirical evidence available to support this proposition. Indeed, overestimation is also possible due to primary selection bias if the competition for jobs with regular workhours is harsh, and shift work remains the only available option for those with less attractive competencies and worse health. In the future, research on selection into shift work and day work is needed.

Our target population was homogeneous in terms of gender and occupational group. This homogeneity may be both a strength and limitation of this study. The increased risk of CVD among shift workers was originally shown in a sample of female nurses (5) (ie, a population similar to that used in this study). Thus the similarity of the study sample suggests that our findings apply to populations in which shift-work-related CVD risk has been demonstrated. Socioeconomic position has been suggested as a major source of confounding in shift work research (9), and, because there was no variation in socioeconomic position in this study, this source of confounding was eliminated. However, findings on female nurses are not necessarily generalizable to other occupational groups or to men; thus replications with other populations are needed.

In conclusion, this prospective study with a 2- to 4year follow-up suggests that selection out of shift work is an unlikely source of major bias in research on shift work and CVD.

\section{Acknowledgments}

This study was supported by the Finnish Work Environment Fund and the Academy of Finland (projects 104891, 105195 and 110451).

\section{References}

1. Boggild H, Knutsson A. Shift work, risk factors and cardiovascular disease [review]. Scand J Work Environ Health.
1999;25(2):85-99.

2. Knutsson A, Boggild H. Shiftwork and cardiovascular disease: review of disease mechanisms. Rev Environ Health. 2000;15(4):359-72.

3. Taylor PJ, Pocock SJ. Mortality of shift and day workers 1956-68. Br J Ind Med. 1972;29(2):201-7.

4. Tenkanen L, Sjöblom T, Kalimo R, Alikoski T, Härmä M. Shift work, occupation and coronary heart disease over 6 years of follow-up in the Helsinki Heart Study. Scand J Work Environ Health. 1997;23(4):257-65.

5. Kawachi I, Colditz GA, Stampfer MJ, Willett WC, Manson JE, Speizer FE, et al. Prospective study of shift work and risk of coronary heart disease in women. Circulation. 1995; 92(11):3178-82.

6. Knutsson A, Åkerstedt T, Jonsson BG, Orth-Gomer K. Increased risk of ischaemic heart disease in shift workers. Lancet. 1986;2(8498):89-92.

7. Tuchsen F. Working hours and ischaemic heart disease in Danish men: a 4-year cohort study of hospitalization. Int $\mathrm{J}$ Epidemiol. 1993;22(2):215-21.

8. Virtanen SV, Notkola V. Socioeconomic inequalities in cardiovascular mortality and the role of work: a register study of Finnish men. Int J Epidemiol. 2002;31(3):614-21.

9. Boggild H, Suadicani P, Hein HO, Gyntelberg F. Shift work, social class, and ischaemic heart disease in middle aged and elderly men; a 22 year follow up in the Copenhagen Male Study. Occup Environ Med. 1999;56(9):640-5.

10. Carpenter LM. Some observations on the healthy worker effect. Br J Ind Med. 1987;44(5):289-91.

11. Zenz C, Dickerson OB, Horvath EP. Occupational medicine. St Louis (MO): Mosby; 1994.

12. Morikawa Y, Nakagawa H, Miura K, Ishizaki M, Tabata M, Nishijo M, et al. Relationship between shift work and onset of hypertension in a cohort of manual workers. Scand J Work Environ Health. 1999;25(2):100-4.

13. Knutsson A. Methodological aspects of shift-work research. Chronobiol Int. 2004;21(6):1037-47.

14. Kivimäki M, Kuisma P, Virtanen M, Elovainio M. Does shift work lead to poorer health habits? a comparison between women who had always done shift work with those who had never done so. Work Stress. 2001;15:3-13.

15. Koskenvuo M, Kaprio J, Rose RJ, Kesäniemi A, Sarna S, Heikkilä K, et al. Hostility as a risk factor for mortality and ischemic heart disease in men. Psychosom Med. 1988; 50(4):330-40.

16. Haapanen N, Miilunpalo S, Pasanen M, Oja P, Vuori I. Agreement between questionnaire data and medical records of chronic diseases in middle-aged and elderly Finnish men and women. Am J Epidemiol. 1997;145(8):762-9.

Received for publication: 27 December 2005 